12. Kinsler VA, Chong WK, Aylett SE et al (2008) Complications of congenital melanocytic naevi in children: analysis of 16 years experience and clinical practice. Br J Dermatol 159:907-914

13. Koot HM, Waard-van der Spek de, Peer CD et al (2000) Psychosocial sequelae in 29 children with giant congenital melanocytic naevi. Clin Exp Dermatol 25:589-593

14. Krengel S (2005) Nevogenesis - new thoughts regarding a classical problem. Am J Dermatopathol 27:456-465

15. Krengel S, Hauschild A, Schafer T (2006) Melanoma risk in congenital melanocytic naevi: a systematic review. Br J Dermatol 155:1-8

16. Krengel $\mathrm{S}$, Breuninger $\mathrm{H}$, Hauschild $A$ et al (2008) Installation of a network for patients with congenital melanocytic nevi in German-speaking countries. J Dtsch Dermatol Ges 6:204-208

17. Krengel $\mathrm{S}$, Breuninger $\mathrm{H}$, Beckwith $\mathrm{M}$ et al (2011) Meeting report from the 2011 international expert meeting on large congenital melanocytic nevi and neurocutaneous melanocytosis, Tuebingen. Pigment Cell Melanoma Res 24:E1-6

18. Mahe E, Beauchet A, Paula CM de et al (2011) Outdoor sports and risk of UV-related skin lesions in children: evaluation of risks, and prevention. $\mathrm{Br}$ J Dermatol 165:360-367

19. Makkar HS, Frieden IJ (2004) Neurocutaneous melanosis. Semin Cutan Med Surg 23:138-144

20. Marghoob AA, Schoenbach SP, Kopf AW et al (1996) Large congenital melanocytic nevi and the risk for the development of malignant melanoma. A prospective study. Arch Dermatol 132:170-175

21. Marghoob AA, Dusza S, Oliveria S et al (2004) Number of satellite nevi as a correlate for neurocutaneous melanocytosis in patients with large congenital melanocytic nevi. Arch Dermatol 140:171-175

22. Puzenat E (2010) Photoprotection and children: why and how? Arch Pediatr 17:914-915

23. Siskind V, Hughes MC, Palmer JM et al (2011) Nevi, family history, and fair skin increase the risk of second primary melanoma. J Invest Dermatol 131:461-467

24. Stierman SC, Tierney EP, Shwayder TA (2009) Halo congenital nevocellular nevi associated with extralesional vitiligo: a case series with review of the literature. Pediatr Dermatol 26:414-424

25. Streams BN, Lio PA, Mihm MC et al (2004) A nonepidermal, primary malignant melanoma arising in a giant congenital melanocytic nevus 40 years after partial surgical removal. J Am Acad Dermatol 50:789-792

26. Tromberg J, Bauer B, Benvenuto-Andrade $C$ et al (2005) Congenital melanocytic nevi needing treatment. Dermatol Ther 18:136-150

27. Wiecker TS, Luther H, Buettner P et al (2003) Moderate sun exposure and nevus counts in parents are associated with development of melanocytic nevi in childhood: a risk factor study in 1,812 kindergarten children. Cancer 97:628-638

28. Zaal LH, Mooi WJ, Sillevis Smitt JH et al (2004) Classification of congenital melanocytic naevi and malignant transformation: a review of the literature. Br J Plast Surg 57:707-719

29. Zalaudek I, Guelly C, Pellacani G et al (2011) The dermoscopical and histopathological patterns of nevi correlate with the frequency of $B R A F$ mutations. J Invest Dermatol 131:542-545

30. Zedek DC, McCalmont TH (2011) Spitz nevi, atypical spitzoid neoplasms, and spitzoid melanoma. Clin Lab Med 31:311-320

Monatsschr Kinderheilkd 2011 • 159:1084 DOI 10.1007/s00112-011-2535-y

C Springer-Verlag 2011

M. Wabitsch · B. Koletzko · A. Moß

Ernährungskommission der Deutschen Gesellschaft

für Kinder- und Jugendmedizin e.V, Berlin

\title{
Erratum zu: Vitamin-D-Versorgung im Säuglings-, Kindes- und Jugendalter
}

\section{Kurzfassung der Stellungnahme der Ernährungskommission der Deutschen Gesellschaft für Kinder- und Jugendmedizin (DGKJ) in Zusammenarbeit mit der Arbeitsgemeinschaft Pädiatrische Endokrinologie (APE)}

Monatsschrift Kinderheilkunde 159:766-774.
http://dx.doi.org/10.1007/s00112-011-2407-5

Die Angaben zu Autorschaft und Korrespondenzadresse dieses Beitrags waren fehlerhaft. Die korrekten Angaben lauten: Autoren:

Ernährungskommission der Deutschen Gesellschaft für Kinder- und Jugendmedizin $(D G K J)^{*}$ in Zusammenarbeit mit der Arbeitsgemeinschaft Pädiatrische Endokrinologie

*(Böhles HJ, Fusch C, Genzel-Boroviczény $\mathrm{O}$, Jochum F, Kauth T, Kersting $\mathrm{M}$, Koletzko B (Vorsitzender), Lentze MJ, Moß A (Gast), Mihatsch WA, Przyrembel H, Schnabel D (Gast), Wabitsch M)

Korrespondenzadresse:

Ernährungskommission der Deutschen Gesellschaft für Kinderund Jugendmedizin e.V. Chausseestraße 128/129, 10115 Berlin

Prof. Dr. M. Wabitsch

(korrespondierender Autor)

info@dgkj.de
Der Hinweis in der Fußnote auf ausführliche Informationen zu Physiologie, Pathophysiologie und zu klinischen Studien unter http://www.dgkj.de entfällt.

Wir bitten, zukünftig die korrekten Angaben zu verwenden und den Fehler zu entschuldigen.

\section{Korrespondenzadresse \\ Prof. Dr. M. Wabitsch}

Ernährungskommission der Deutschen Gesellschaft für Kinder- und Jugendmedizin e.V. Chausseestr. 128/129, 10115 Berlin info@dgkj.de 